\section{Translational and clinical research highlights from the 38th San Antonio Breast Cancer Symposium}

\author{
Caterina Fontanella, ${ }^{1,2}$ \&abio Puglisi ${ }^{* 1,2}$
}

38th San Antonio Breast Cancer Symposium, San Antonio, TX, USA, 8-12 December 2015

In November 1978, during Breast Cancer Awareness Week, the first annual San Antonio Breast Cancer Symposium (SABCS) was held as a part of an educational program conceived to reduce breast cancer death rate. Only 141 physicians attended the conference. Recently, the 38th edition of the SABCS welcomed $>7000$ attendees from all over the world. We will discuss a selection of the most significant advances presented at the 2015 SABCS, specifically focusing on those data that could have an impact on the clinical practice in the near future.

First draft submitted: 11 January 2016; Accepted for publication: 20 January 2016; Published online: 9 February 2016

\section{Circulating tumor cells predict poor survival: a SUCCESSful project}

High-risk breast cancer (BC) patients (node positive or T2-T4 or grade 3 or women $\leq 35$ years or hormone receptor $[\mathrm{Hr}]$ negative) were enrolled in the SUCCESS A trial [1]. This Phase III study randomized 3745 patients to adjuvant fluorouracil-epirubicin-cyclophosphamide followed by docetaxel or gemcitabine-docetaxel. In addition, patients were randomized to 2 versus 5 years of zoledronic acid. Circulating tumor cells (CTCs) measurements were available for 1103 patients. Multivariate analysis showed significant independent prognostic role for CTC status 2 years after chemotherapy on overall survival (OS; hazard ratio [HR]: 3.95; 95\% CI: 2.13-7.32; $<<0.001$ ) and disease-free survival (DFS; HR: 2.28; 95\% CI: 1.48-3.50; $p<0.001$ ), independently from hormone- and HER2-receptor status. In daily practice, CTC testing after curative BC treatment may be used as a surveillance marker to identify patients at higher risk for relapse who could benefit from an intensified follow-up program or secondary treatment intervention [2].

\section{HER2-positive tumors: a long, heterogeneous story}

After the introduction in clinical practice of the monoclonal antibody trastuzumab, the natural history of patients diagnosed with HER2-positive BC has dramatically changed. Over the last decade, new insights have been discovered on the HER2 pathway and new HER2-targeted drugs have been developed [3].

A whole-exome sequencing analysis, performed on 203 patients enrolled in the NeoALLTO trial [4], demonstrated that mutation of PIK3CA was the strongest predictor of response to HER2-targeted therapies (odds ratio for absence of pathological complete response [pCR]: 0.42 ; $\mathrm{p}=0.019$ ) [5]. Moreover, the study showed 33 pathways significantly associated with response; in the

'Department of Biological \& Medical Sciences, University of Udine, Piazzale Kolbe, 4 - 33100 Udine, Italy

2Department of Oncology, University Hospital of Udine, Piazzale S M Misericordia, 33100 Udine, Italy

*Author for correspondence: Tel.: +39 0432 552754/0432 559309; Fax: +39 0432 552762; fabio.puglisi@uniud.it

\section{KEYWORDS}

- 38th SABCS • breast cancer • clinical research

- translational research 
trastuzumab arm, 23 of the 33 pathways showed an association with response but none was independent of PIK3CA mutation. Considering that genes involved in the response to HER2targeted drugs are mainly connected through the PIK3CA gene, and analysis of PIK3CA status in HER2-positive BC patients may be of great importance in daily practice.

Moving research from the laboratory to the clinic, other important results have been presented at the symposium.

The BCIRG-006 trial [6] evaluated three different adjuvant regimens in 3222 HER2positive patients: four cycles of doxorubicin/ cyclophosphamide followed by four cycles of docetaxel (AC-T) versus four cycles of doxorubicin/cyclophosphamide followed by four cycles of docetaxel and 1 year of trastuzumab (AC-TH) versus six cycles of docetaxel plus carboplatin and 1 year of trastuzumab (TCH). After a median follow-up of 10.3 years, $28 \%$ of risk of disease recurrence reduction was observed in the AC-TH arm (HR: 0.49; 95\% CI: 0.37-0.65; p < 0.001) and $23 \%$ of reduction in the TCH arm (HR: 0.61; 95\% CI: 0.47-0.79; $\mathrm{p}=0.002)$, compared with AC-T arm [7]. Moreover, a significant OS benefit was also seen in both trastuzumab-containing arms compared with AC-T (risk of death reduction: $37 \%$ for AC-TH and $24 \%$ for TCH). To note, the anthracyclines/trastuzumab containing strategy showed a fivefold higher number of congestive heart failure cases compared with anthracyclines-free strategy (21 cases with AC-TH vs four cases with TCH). Considering that the $\mathrm{TCH}$ regimen showed to be a very active regimen, which essentially eliminates the cardiotoxicity issues, it may be considered as the first choice for patients with cardiac concerns.

In the Phase III TH3RESA trial [8], 602 patients with HER2-positive metastatic breast cancer who progressed after previous treatment with trastuzumab and lapatinib were randomized to receive HER2-targeted antibody-drug conjugate ado-trastuzumab emtansine (T-DM1) or treatment of physician's choice (TPC). After a median follow-up of 30.5 months, the median OS was 22.7 months in the 404 patients assigned to T-DM1 compared with 15.8 months in the 198 patients assigned to TPC (HR: $0.68 ; 95 \% \mathrm{CI}$ : $0.54-0.85 ; \mathrm{p}=0.0007)$, regardless of patient's age, $\mathrm{Hr}$-status, visceral involvement and number of prior therapeutic lines [9]. Moreover, the incidence of grade 3 or higher adverse events was 7.3\% higher among patients assigned to TPC.
The efficacy of T-DM1 was also tested in the neoadjuvant setting for HER2-positive, Hr-positive BC in the WSG-ADAPT HER 2+/Hr+ Phase II trial. Overall, 376 patients were randomized to 12 weeks of neoadjuvant therapy with: T-DM1 alone, T-DM1 with endocrine therapy (ET; according to menopausal status) or trastuzumab plus ET [10]. After surgery, standard TPC and completion of 1 year trastuzumab were recommended. The pCR rate was $40.5 \%$ with T-DM1, 45.8\% with T-DM1/ET and 6.7\% with trastuzumab/ET $(\mathrm{p}<0.001$ for difference between T-DM1 arms versus trastuzumab arm). Notably, the $15.8 \%$ of patients who harbored a PIK3CA mutation achieved a lower pCR rate (35.4\% for wild-type and $17.6 \%$ for mutated).

These studies showed that T-DM1 has a key role in the treatment strategy of HER2-positive disease, in both early and metastatic setting.

\section{Carboplatin for triple-negative breast cancer: an old drug with a new dress on}

It has been demonstrated that up to $20 \%$ of triplenegative breast cancer (TNBC) patients harbor germline $B R C A$ mutations and that BRCA mutations carriers may be more sensitive to platinumbased chemotherapy [11]. However, little is still known about the association between mutations and response to nonplatinum-based therapy.

A next-generation sequencing analysis was conducted on blood samples of TNBC patients exposed to fluorouracil-epirubicin-cyclophosphamide with or without bevacizumab enrolled in the neoadjuvant GeparQuinto study [12,13]. The analysis showed that patients harboring $B R C A 1 / 2$ mutations had a higher pCR rate compared with patients without mutations $(50.0$ vs $31.1 \%$, respectively, $\mathrm{p}=0.002$ ), but no significant impact of $\mathrm{pCR}$ on DFS was detected in mutations carriers (HR: 0.48; 95\% CI: 0.18-1.27; $\mathrm{p}=0.129)$. These data suggest that TNBC patients with $B R C A$ mutations may experience a better response when exposed to standard neoadjuvant treatment. However, the effect of pCR on prognosis is not demonstrated among the mutation carriers.

At the 2015 San Antonio Breast Cancer Symposium (SABCS), the updated survival analysis of two large neoadjuvant trials confirmed the beneficial effect of adding carboplatin to anthracycline/taxane-based chemotherapy in TNBC.

In the GeparSixto trial [14], patients were treated for 18 weeks with weekly paclitaxel plus 
nonpegylated-liposomal doxorubicin. They were randomly assigned (1:1 ratio) to receive concomitant weekly carboplatin or not. In addition, the 315 women with TNBC received concurrently bevacizumab until surgery, and the 273 women with HER2-positive cancer received concurrently trastuzumab and lapatinib. After a median follow-up of 28 months, in the TNBC cohort, the addition of carboplatin lead to a $9.7 \%$ higher 3-year DFS rate (85.8\% with carboplatin vs $76.1 \%$ without carboplatin; HR: 0.59; 95\% CI: 0.33-0.96; $\mathrm{p}=0.035)$; no effect of carboplatin was noticed in the HER2-positive cohort $(p=0.372)$ [15]. In contrast, the updated analysis of the CALGB (Alliance) 40603 trial [16] showed only a nonsignificant improvement in 3-year event-free survival (EFS) rate by the addition of carboplatin to 12 courses of weekly paclitaxel followed by doxorubicin/cyclophosphamide every 2 weeks for four cycles $(76.5 \%$ with carboplatin vs $71.6 \%$ without carboplatin; HR: $0.84 ; 95 \% \mathrm{CI}$ : $0.58-1.22 ; \mathrm{p}=0.36)$ [17]. However, the CALGB study was not powered to assess the impact of carboplatin on time-to-event end points.

To note, in both trials no differences in DFS/ event-free survival were detected by the addition of bevacizumab.

The effect of carboplatin in neoadjuvant setting was also explored in the TNBC cohort of the WSG-ADAPT trial. Overall, 336 earlystage TNBC patients were randomized to 12 weeks of nab-paclitaxel/gemcitabine or nab-paclitaxel/carboplatin. Interim analysis, performed on 130 patients, showed a higher pCR rate in the platinum-based arm compared with platinum-free arm (49.2 vs $25.0 \%$, respectively; $\mathrm{p}=0.006$ ) [18]. Interestingly, early response, defined as $<500 \mathrm{BC}$ cells and/or Ki-67 decrease $\geq 30 \%$ in 3-week biopsy, was positively associated with pCR rate ( $44.4 \%$ in responders vs $19.5 \%$ in nonresponders; $\mathrm{p}<0.001)$.

Taken together, these results support the development of neoadjuvant strategies designed to further assess the value of platinum-based regimens in early-stage TNBC in daily practice. However, a better characterization of TNBC, especially in terms of $B R C A$ status, is mandatory for future research.

\section{Which news for Hr-positive/HER2-negative} $\mathrm{BC}$ patients?

The anti-PD-1 pembrolizumab has been tested in PD-L1-positive, $\mathrm{Hr}$-positive/HER2-negative with heavily pretreated metastatic breast cancer in the KEYNOTE-028 multicohort, Phase Ib study. Among the 25 patients enrolled, three patients achieved a partial response and four patients a stable disease, with a median time to response of 8.0 weeks and a median duration of stable disease of 16 weeks [19]. Only four patients experienced grade 3-4 adverse events, confirming the safety of pembrolizumab and endorsing the opportunity to further investigate this agent.

The ABCSG-18 trial randomized 3425 postmenopausal patients with $\mathrm{Hr}$-positive $\mathrm{BC}$ receiving adjuvant aromatase inhibitors to the addition of the anti-RANK-ligand denosumab or placebo. Outcome results, presented at the 2015 SABCS after a median follow-up of 4 years, showed a significant impact of denosumab in terms of DFS (HR: 0.816; $\mathrm{p}=0.051$; DFS difference between the two arms was $4 \%$ at 2 years, $7 \%$ at 5 years and $10 \%$ at 7 years; $\mathrm{p}=0.0163$ [20]. Considering the previously reported dramatic reduction of bone-related events (HR for fracture: 0.5; $\mathrm{p}<0.0001$ ) [21], denosumab is going to gain an important role in the adjuvant setting.

Between 1977 and 1983, the Danish Breast Cancer Cooperative Group 77B (DBCG-77B) trial randomized $1146 \mathrm{Hr}$-positive high-risk premenopausal women (node positive or tumor $>5 \mathrm{~cm}$ ) to receive: oral cyclophosphamide, cyclophosphamide-methotrexate-fluorouracil, levamisole or observation alone [22]. The analysis of the 10-year DFS according to breast cancer subtypes presented at the 2015 SABCS showed that patients with luminal A BC did not benefit from chemotherapy (HR: 1.07; 95\% CI: 0.53-2.14; $\mathrm{p}=0.86)$, whereas patients with nonluminal A subtypes did (HR: 0.50; 95\% CI: 0.38-0.66; $\mathrm{p}<0.001)$. This heterogeneity was statistically significant $(p=0.048)$ [23]. These results further support the approach of avoiding adjuvant chemotherapy in patients with luminal A BC, even in presence of large tumors or nodal involvement.

\section{Conclusion}

The SABCS offered the unique opportunity to bring together the leading results from international studies. New and compelling data for the use of targeted therapies, as well as new insights on the use of traditional chemotherapy in both adjuvant and metastatic settings were presented and discussed, increasing the knowledge about $\mathrm{BC}$ and consequently improving every day clinical practice. 
Financial \& competing interests disclosure The authors have no relevant affiliations or financial involvement with any organization or entity with a financial interest in or financial conflict with the subject matter or materials discussed in the manuscript. This includes employment, consultancies, honoraria, stock ownership or options, expert testimony, grants or patents received or pending, or royalties.

No writing assistance was utilized in the production of this manuscript.

\section{References}

1 Janni W, Rack B, Fasching P et al. Persistence of circulating tumor cells in high risk early breast cancer patients during follow-up care suggests poor prognosis - results from the adjuvant SUCCESS A trial. Presented at: San Antonio Breast Cancer Symposium 2015. San Antonio, TX, USA, 8-12 December 2015.

2 Puglisi F, Fontanella C, Numico G et al. Follow-up of patients with early breast cancer: is it time to rewrite the story? Crit. Rev. Oncol. Hematol. 91(2), 130-141 (2014).

3 Puglisi F, Fontanella C, Amoroso V et al. Current challenges in HER2-positive breast cancer. Crit. Rev. Oncol. Hematol. 98, 211-221 (2016).

4 Baselga J, Bradbury I, Eidtmann $\mathrm{H}$ et al. Lapatinib with trastuzumab for HER2positive early breast cancer (NeoALTTO): a randomised, open-label, multicentre, Phase 3 trial. Lancet 379(9816), 633-640 (2012).

5 Pusztai L, Shi W, Jiang T et al. Whole exome sequencing of pre-treatment biopsies from the neoALTTO trial to identify DNA aberrations associated with response to HER2-targeted therapies. Presented at: San Antonio Breast Cancer Symposium 2015. San Antonio, TX, USA, 8-12 December 2015.

6 Slamon D, Eiermann W, Robert N et al. Adjuvant trastuzumab in HER2-positive breast cancer. N. Engl. J. Med. 365(14), 1273-1283 (2011).

7 Slamon D, Eirmann W, Robert N et al. Ten year follow-up of the BCIRG-006 trial comparing doxorubicin plus cyclophosphamide followed by docetaxel $\left(\mathrm{AC}^{\circledR} \mathrm{T}\right)$ with doxorubicin plus cyclophosphamide followed by docetaxel and trastuzumab $\left(\mathrm{AC}^{\circledR} \mathrm{TH}\right)$ with docetaxel, carboplatin and trastuzumab (TCH) in HER 2+ early breast cancer patients. Presented at: San Antonio Breast Cancer Symposium 2015. San Antonio, TX, USA, 8-12 December 2015.

8 Krop IE, Kim SB, González-Martín A et al. Trastuzumabemtansine versus treatment of physician's choice for pretreated HER2positive advanced breast cancer (TH3RESA): a randomised, open-label, Phase 3 trial. Lancet Oncol. 15(7), 689-699 (2014).
9 Wildiers H, Kim S-B, Gonzalez-Martin A et al. Trastuzumab emtansine improves overall survival versus treatment of physician's choice in patients with previously treated HER2positive metastatic breast cancer: Final overall survival results from the Phase 3 TH3RESA study. Presented at: San Antonio Breast Cancer Symposium 2015. San Antonio, TX, USA, 8-12 December 2015.

10 Harbeck N, Gluz O, Christgen M et al. Final analysis of WSG-ADAPT HER2+/HR+ Phase II trial: efficacy, safety, and predictive markers for 12-weeks of neoadjuvant TDM1 with or without endocrine therapy versus trastuzumab+endocrine therapy in HER2positive hormone-receptor-positive early breast cancer. Presented at: San Antonio Breast Cancer Symposium 2015. San Antonio, TX, USA, 8-12 December 2015.

11 ASCO post. www.ascopost.com

12 vonMinckwitz G, Loibl S, Untch M et al. Survival after neoadjuvant chemotherapy with or without bevacizumab or everolimus for HER2-negative primary breast cancer (GBG 44-GeparQuinto). Ann. Oncol. 25(12), 2363-2372 (2014).

13 Fasching PA, Loibl S, Eidtmann $\mathrm{H}$ et al. $B R C A$ mutations, therapy response and prognosis in the neoadjuvant GeparQuinto study. Presented at: San Antonio Breast Cancer Symposium 2015. San Antonio, TX, USA, 8-12 December 2015.

14 vonMinckwitz G, Eidtmann H, Rezai M et al. Neoadjuvant chemotherapy and bevacizumab for HER2-negative breast cancer. N. Engl. J. Med. 366(4), 299-309 (2012).

15 vonMinckwitz G, Loibl S, Schneeweiss A et al. Early survival analysis of the randomized Phase II trial investigating the addition of carboplatin to neoadjuvant therapy for triple-negative and HER2-positive early breast cancer (GeparSixto). Presented at: San Antonio Breast Cancer Symposium 2015. San Antonio, TX, USA, 8-12 December 2015.

16 Sikov WM, Berry DA, Perou CM et al. Impact of the addition of carboplatin and/or bevacizumab toneoadjuvant once-per-week paclitaxel followed by dose-dense doxorubicin and cyclophosphamide on pathologic complete response rates in stage II to III. triple-negative breast cancer: CALGB 40603 (Alliance). J. Clin. Oncol. 33(1), 13-21 (2015).

17 Sikov WM, Berry DA, Perou CM et al. Event-free and overall survival following neoadjuvant weekly paclitaxel and dose-dense $\mathrm{AC}+$ /- carboplatin and/or bevacizumab in triple-negative breast cancer: outcomes from CALGB 40603 (Alliance). Presented at: San Antonio Breast Cancer Symposium 2015. San Antonio, TX, USA, 8-12 December 2015.

18 Gluz O, Nitz U, Liedtke C, Christgen $\mathrm{M}$ et al. Comparison of 12 weeks neoadjuvant nab-paclitaxel combined with carboplatinum vs. gemcitabine in triple-negative breast cancer: WSG-ADAPT TN randomized Phase II trial. Presented at: San Antonio Breast Cancer Symposium 2015. San Antonio, TX, USA, 8-12 December 2015.

19 Rugo H, Delord J, Im S et al. Preliminary efficacy and safety of pembrolizumab (MK-3475) in patients with PD-L1-positive, estrogen receptor-positive (ER+)/HER2negative advanced breast cancer enrolled in KEYNOTE-028. Presented at: San Antonio Breast Cancer Symposium 2015. San Antonio, TX, USA, 8-12 December 2015.

20 Gnant M, Pfeiler G, Dubsky PC et al. The impact of adjuvant denosumab on disease-free survival: results from 3425 postmenopausal patients of the ABCSG-18 trial. Presented at: San Antonio Breast Cancer Symposium 2015. San Antonio, TX, USA, 8-12 December 2015.

21 Gnant M, Pfeiler G, Dubsky PC et al. Adjuvant denosumab in breast cancer (ABCSG-18): a multicentre, randomised, double-blind, placebo-controlled trial. Lancet 386(9992), 433-443 (2015).

22 Ejlertsen B, Mouridsen HT, Jensen MB et al. Cyclophosphamide, methotrexate, and fluorouracil; oral cyclophosphamide; levamisole; or no adjuvant therapy for patients with high-risk, premenopausal breast cancer. Cancer 116(9), 2081-2089 (2010).

23 Nielsen TO, Jensen M-B, Gao D et al. High risk premenopausal luminal A breast cancer patients derive no benefit from adjuvant chemotherapy: results from DBCG77B randomized trial. Presented at: San Antonio Breast Cancer Symposium 2015. San Antonio, TX, USA, 8-12 December 2015. 